\title{
Bone remodelling around a cementless glenoid component
}

\author{
Daniel R. Suárez • Harrie Weinans • Fred van Keulen
}

Received: 30 April 2011 / Accepted: 5 November 2011 / Published online: 23 November 2011

(C) The Author(s) 2011. This article is published with open access at Springerlink.com

\begin{abstract}
Post-operative change in the mechanical loading of bone may trigger its (mechanically induced) adaptation and hamper the mechanical stability of prostheses. This is especially important in cementless components, where the final fixation is achieved by the bone itself. The aim of this study is, first, to gain insight into the bone remodelling process around a cementless glenoid component, and second, to compare the possible bone adaptation when the implant is assumed to be fully bonded (best case scenario) or completely loose (worst case scenario). 3D finite element models of a scapula with and without a cementless glenoid component were created. 3D geometry of the scapula, material properties, and several physiological loading conditions were acquired from or estimated for a specific cadaver. Update of the bone density after implantation was done according to a node-based bone remodelling scheme. Strain energy density for different
\end{abstract}

D. R. Suárez $(\varangle)$

F. de Ingeniería, Pontificia Universidad Javeriana,

Bogotá, Colombia

e-mail: d-suarez@javeriana.edu.co

D. R. Suárez

Department of Orthopaedics, Leiden University MC,

Leiden, The Netherlands

D. R. Suárez · F. van Keulen

Precision and Microsystems Engineering, 3mE Faculty,

Delft University of Technology, Mekelweg 2,

2628 CD Delft, The Netherlands

H. Weinans

Orthopaedic Research Laboratory, Erasmus MC, P.O. Box 2040,

3000 CA Rotterdam, The Netherlands loading conditions was evaluated, weighted according to their frequencies in activities of daily life and used as a mechanical stimulus for bone adaptation. The average bone density in the glenoid increased after implantation. However, local bone resorption was significant in some regions next to the bone-implant interface, regardless of the interface condition (bonded or loose). The amount of bone resorption was determined by the condition imposed to the interface, being slightly larger when the interface was loose. An ideal screw, e.g. in which material fatigue was not considered, was enough to keep the interface micromotions small and constant during the entire bone adaptation simulation.

Keywords Glenoid · Bone remodelling .

Cementless implant · Finite element analysis · Shoulder

\section{Introduction}

Total shoulder arthroplasty (TSA) is the replacement of the glenohumeral joint with a prosthesis consisting of a humeral and a glenoid component. It is performed as a salvage procedure to reduce pain and regain range of motion of the shoulder after severe arthritis, trauma or poor results from a previous shoulder surgery. Although the outcome of TSA is satisfactory, there are still significant problems, mostly related to the glenoid component (Bohsali et al. 2006; Torchia et al. 1997; Wirth and Rockwood 1996), making this surgical procedure less predictable than hip or knee joint replacements (Bohsali et al. 2006).

Morphological changes in bone around prostheses are often observed and may threaten their long-term fixation. The modified loading condition in the host bone due to the presence of the implant is highly responsible for these 
changes (Frost 1988; Huiskes et al. 1987; van Rietbergen et al. 1993; Weinans et al. 1993). This change in the way bone is locally loaded is commonly known as stress shielding, and it produces an adaptive bone remodelling (Frost 1988; Huiskes et al. 1987). This adaptation has been extensively studied for femoral stems in total hip arthroplasty, but its relevance in the clinical outcome of a TSA is largely unknown (Pelletier et al. 2008).

Numerical studies have already reported a possible bone adaptation around glenoid prostheses (Gupta et al. 2004a,b; Lacroix et al. 2000; Orr et al. 1988; Stone et al. 1999); however, they based their predictions on the stress shielding found right after implantation of a glenoid component without explicitly modelling bone adaptation. Prediction of those previous studies was then limited to the presence or absence of stress shielding, but the extension and dynamics of the bone adaptation process were not addressed. In general, finite element (FE) analysis has played an important role in those and other studies that focused on bone adaptation (Hart 2001), studying hypothetical cases in less time than clinical trails, and studying the effect of stress shielding without the presence of other factors that also induce changes in bone.

Adaptive bone remodelling may compromise the quality of the implant's bone bed in the long term after TSA. Therefore, the aim of this study is, first, to gain insight into the bone remodelling process around a cementless glenoid component, and second, to compare the possible bone adaptation when the bone-implant interface is assumed to be fully bonded (best case scenario) and completely loose (worst case scenario). With this goal in mind, a previously presented 3D FE model (Suárez et al. 2009a,b) was used in combination with a site-specific, node-based, bone remodelling algorithm (Jacobs et al. 1995). The impact of bone adaptation in the glenoid was assessed by the change of bone density over time, locally and throughout the entire glenoid. Changes in local Young modulus, (maximum and minimum) bone principal strains and, in the case of a loose interface, the bone-implant interface micromotions were also evaluated.

\section{Methods}

\subsection{The FE model}

3D geometry, bone properties, and muscle and joint forces during arm abduction and forward flexion were acquired through a computed tomography (CT) data set and a musculoskeletal model of a single scapula. This process has been previously described (Suárez et al. 2009a,b), and it is summarized here.

Geometry and local material properties of the scapula were obtained from a CT data set (with a voxel size of $\left.0.3 \times 0.3 \times 0.5 \mathrm{~mm}^{3}\right)$ of a scapula retrieved from a cadaver (man: 90 years, $73 \mathrm{~kg}, 173 \mathrm{~cm}$ ) in a previous study made by Veeger et al. (1991). The shoulder of the cadaver did not present signs of trauma or degeneration. Contours of the scapula were extracted, and triangular elements were defined to form a surface model using the techniques described by Boissonnat (1988).

The Young's modulus, $E$ [MPa], and the apparent density of the bone, $\rho_{a}\left[\mathrm{~g} / \mathrm{cm}^{3}\right]$, were obtained through their relationship with the CT Hounsfield units [HU] in the same CT data set. The $\rho_{a}-\mathrm{HU}$ relationship, assessed using an object with known density (Plexiglas) during image acquisition, was:

$\rho_{a}=1063+1.1012 \mathrm{HU}$.

To quantify the Young's modulus for each element, two $E-\rho_{a}$ relationships were used, one for cancellous bone (Rice et al. 1988):

$E=60+900 \rho_{a}^{2}$

if $\rho_{a} \leq 1.54 \mathrm{~g} / \mathrm{cm}^{3}$, and a second one for cortical bone (Schaffler and Burr 1988):

$E=90 \rho_{a}^{7.4}$

if $\rho_{a}>1.54 \mathrm{~g} / \mathrm{cm}^{3}$.

An averaging procedure was applied to avoid numerical problems due to large stiffness differences between neighbouring elements in the cancellous bone. The apparent density for every element was the result of averaging the voxel values inside a representative volume element (RVE) that encloses the element and then applying the given $E-\rho$ relationships. The characteristic length of the RVE (LRVE) should be at least five intertrabecular lengths to fulfil the continuum assumption for cancellous bone (Hollister et al. 1994; van Rietbergen and Huiskes 2001). After a preliminary test, in which the LRVE was modified from 1.7 and $7 \mathrm{~mm}$, a value of $5 \mathrm{~mm}$ was chosen.

The resultant bone density ranged from 0.105 and $2 \mathrm{~g} / \mathrm{cm}^{3}$. The resultant Young's moduli ranged from 70 to 2,200 MPa for cancellous bone and from 2,200 to 15,200 MPa for cortical bone. While the general distribution of bone density and Young's modulus agree with experimental studies, their values are higher than the ones reported in the same studies (Kalouche et al. 2010; Lehtinen et al. 2004). However, the maximal local bone density and Young's modulus are similar to the ones estimated and used in previous numerical models of the scapula (Couteau et al. 2001; Gupta et al. 2004a). Furthermore, the elements along the surface of the scapula were considered to be cortical bone, meaning that the Young's modulus was set to 2,200 MPa in surface elements with initially smaller modulus. This step artificially increased the average bone density of the scapula, but was necessary to clearly define a cortical shell in the entire scapula. Poisson's 


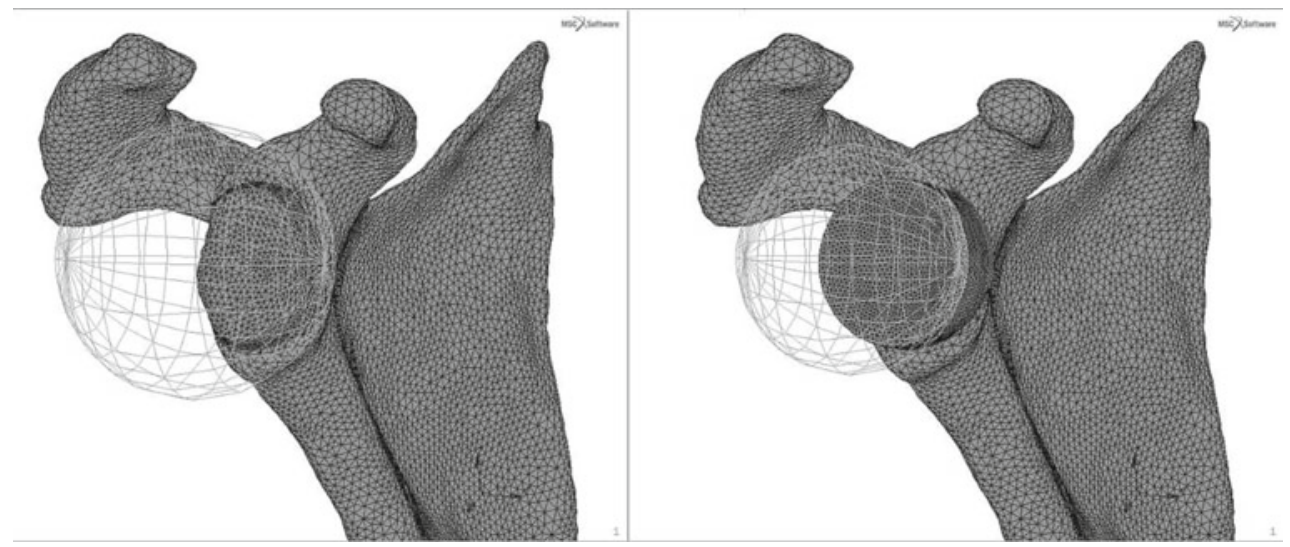

Fig. 1 Finite element model of the scapula. Left intact scapula before TSA. Right scapula with a cementless glenoid component

ratio was set to 0.3 for the complete scapula. The scapula was modelled as an isotropic linear elastic solid.

Virtual implantation of the cementless glenoid component and a single fixation screw (Multiplex, ESKA Implants, Germany) into the scapula was done using a visualization package, DeVIDE (Botha and Post 2008) and the GNU Triangulated Surface Library (GTS) (Popinet and Jones 2006). The implant positioning was approved by a senior surgeon and co-author of this study. The solid mesh of the scapula was created with MSC.Patran 2008r1 (MSC software, USA). The finite element model of the scapula with a glenoid component consisted of approximately 310,000 linear tetrahedral elements.

The cementless glenoid component had a metal back with a porous coating made of a CoCrMo alloy, a polyethylene (PE) inlay and a single central screw (Multiplex, ESKA implants, Germany). The porous coating was simulated as a continuous layer of material with apparent material properties equivalent to the actual properties of the coating. A perfect fit between the metal back and the bone was assumed. The Young's moduli of the metal back and the porous coating were 225 and $3.5 \mathrm{GPa}$, respectively, both with a Poisson's ratio of 0.3 . The PE inlay had a Young's modulus equal to $1.17 \mathrm{GPa}$ and Poisson's ratio of 0.46 . The central screw was simulated as a solid cylinder made of CoCrMo alloy and was fully fixed to implant and bone.

The Delft Shoulder and Elbow Model (DSEM) (van der Helm 1994), which is based on the same scapula, was used to determine all the physiological muscle and joint forces (magnitude, orientation, and application points) on the scapula during arm abduction and forward flexion from $0^{\circ}$ to $90^{\circ}$. In total, eight different arm positions or loading conditions were considered. When the application points defined in the DSEM did not correspond with the nodes in the FE model, the forces were shifted to the nearest nodes and slightly altered according to a minimization formulation in order to retain equilibrium. The glenohumeral joint reaction force was applied by a conforming rigid sphere simulating a prosthetic humeral head, see Fig. 1. From one to three degrees of freedom were constrained at three points of the scapula (the trigonum spinae, the angulus inferior and the most superior point of the acromion) to avoid rigid body motions. The reaction forces at these points were always very small.

\subsection{Interface condition and model solution}

The bone-implant interface was considered fully bonded in a first model (bonded interface) and theoretically represents an ideal interface, in which bone ingrowth had succeeded in the entire interface. In a second FE model (loose interface), the interface was considered completely loose, representing an extreme case of absence of bone ingrowth. In the former case, a Coulomb friction model was used at the bone-implant interface with a coefficient of friction $(\mathrm{CoF})$ equal to 0.65 , which is a conservative value taken from a previous work (Zhang et al. 1999). The influence of the CoF value on the interface micromotions for an identical configuration, except for the screw representation that was modelled as a simple glued area, was done in a previous study. The interface micromotions did not differ significantly when the CoF was modified between 0.5 and 0.9 (Suárez et al. 2009a). The CoF for the interface between the humeral head and the glenoid was set to 0.1 (McKellop et al. 1981) in both models. Mechanical analyses were carried out using a full Newton method (MSC.marc 2008r1, MSC software, USA).

\subsection{The remodelling algorithm}

Bone adaptation after TSA was done according to a bone remodelling, nodal-based iterative scheme proposed by Jacobs et al. (1995). The strain energy density $(U)$ is used as a feedback control variable for the bone density adaptation (Huiskes et al. 1987; Weinans et al. 1993). The reason for choosing this scheme is its numerical stability 
(Jacobs et al. 1995), which preserves anatomically correct features and avoids checker-board patterns that are obtained when element-based algorithms are used. The nodal density change $(\Delta \rho)$ is defined as a function of the local mechanical stimulus $(\psi)$, the available bone surface per unit volume for bone adaptation $(a)$ and simulation time step $(\tau \Delta t)$.

The local mechanical stimulus, $\psi$, is defined as:

$$
\begin{aligned}
& \psi=S-(1+z) S_{r}, \quad \text { if } S>(1+z) S_{r} \\
& \psi=0, \quad \text { if } S_{r}(1-z)<S<(1+z) S_{r} \\
& \psi=S-(1-z) S_{r}, \quad \text { if } S<(1-z) S_{r},
\end{aligned}
$$

where $S[\mathrm{~J} / \mathrm{g}]$ is the remodelling signal, a function of the strain energy density ( $U[\mathrm{MPa}])$ and the apparent density $\left(\rho\left[\mathrm{g} / \mathrm{cm}^{3}\right]\right)$ :

$S=\frac{U}{\rho}$.

This remodelling signal is evaluated at the nodes as an average of the values in the surrounding elements. The constant $z$ defines a threshold (dead) zone for the stimulus, in which the bone remodelling process would not react to a small deviation in the remodelling signal (Frost 1964; Huiskes et al. 1987; Weinans et al. 1993). In this study, $z$ was set to near zero $(<1 \%)$. A local reference value of the remodelling signal, $S_{r}$, was obtained from a reference $\mathrm{FE}$ model of the same scapula before TSA.

It is assumed that bone apposition and resorption can occur only at free bone surfaces, and hence at the periosteal bone surface and inside the bone at the pore surfaces (Martin 1972). Therefore, the available bone surface area per unit volume for bone adaptation, $a\left[\mathrm{~mm}^{2} / \mathrm{mm}^{3}\right]$, is used to decrease the local mechanical stimulus when the local density approaches the minimum or maximum bone density. The value of $a$ is estimated as a function of the bone density using experimental data from Martin (1984),

$$
\begin{aligned}
a(\rho)= & 36.125 v-116.91 v^{2}+181.67 v^{3} \\
& -140.95 v^{4}+40.065 v^{5} \\
v= & \frac{\rho-\rho_{\min }}{\rho_{\max }-\rho_{\min }},
\end{aligned}
$$

where $\rho_{\max }$ and $\rho_{\min }$ are the maximum and minimum bone densities found in the original scapula, i.e. 2.000 and $0.105 \mathrm{~g} / \mathrm{cm}^{3}$, respectively.

In the simulated bone remodelling, the update of the density $(\Delta \rho)$ is carried out in steps of $\tau \Delta t$, which could be considered, regardless the units, as time steps in a 'simulation time scale'. The value of $\tau \Delta t$ was small enough to ensure numerical stability of the remodelling algorithm. The time constant $\tau$ links the simulation time scale with the actual time after implantation in vivo. This time constant can be evaluated comparing the predicted numerical results to in vivo experiments.
The remodelling signal $(S)$ is a linear combination of the remodelling signal for each one of the eight considered arm positions $\left(S_{i}\right)$ that were weighted according to their relative frequencies during activities of daily life as follows,

$S=\sum S_{i} W_{i}$,

where the weighting factors $\left(W_{i}\right)$ are $0.16,0.18,0.12$ and 0.03 for each of the arm elevations at $0^{\circ}, 30^{\circ}, 60^{\circ}$ and $90^{\circ}$, respectively. These values were estimated using records of the arm position during daily activity (Coley et al. 2008). For the first arm position, the weighting factor $\left(W_{1}\right)$ considered all the occurrences (i.e. arm elevations) between 0 and $15^{\circ}$. Weighting factors $W_{2}, W_{3}$ and $W_{4}$ considered the intervals $15-45^{\circ}, 45-75^{\circ}$, and $75^{\circ}$ and higher, respectively.

\subsection{FE model of an intact scapula}

The evaluation of the local mechanical stimulus $(\psi)$ requires an estimation of a reference value for the remodelling signal $\left(S_{r}\right)$, representing the situation before the implantation. Hence, a FE model of the same intact scapula, i.e. without cementless glenoid component, was implemented (see Fig. 1). The material bone properties and loading conditions were acquired in the same way as described previously.

The humeral head case had a radius of $26.6 \mathrm{~mm}$, identical to the one in the cadaver (van der Helm et al. 1992). A layer of cartilage, $1.24 \mathrm{~mm}$ thick in the middle of the glenoid (Yeh et al. 1998) and conforming under loading, was added to the glenoid. Reference values for the remodelling signal $\left(S_{r}\right)$ for different arm positions were weighted in the same way as was done in the FE model with glenoid implant.

Finally, the nodal density update $(\Delta \rho)$ during the bone remodelling process can be written as

$\Delta \rho=\psi \cdot a \cdot \tau \Delta t$

and the updated element density $\left(\rho_{i, t}\right)$ is:

$\rho_{i, t+1}=\rho_{i, t}+\frac{\sum \Delta \rho_{j}}{N}$,

where $i$ is the element number, $t$ is the increment number, $j$ is the node number in the element and $N$ is the number of nodes per element.

\subsection{Average glenoid density, convergence and analysis}

The average glenoid density ( $\left.\rho_{\text {glenoid }}\right)$ during the bone adaptation was calculated as

$\rho_{\text {glenoid }}=\frac{\sum_{i=1}^{n} \rho_{i} V_{i}}{V}$,

where $V$ is the volume of the glenoid, $V_{i}$ is the volume of the element $i$ and $n$ is the number of elements. The bone 
Fig. 2 Bone remodeling scheme used in this study

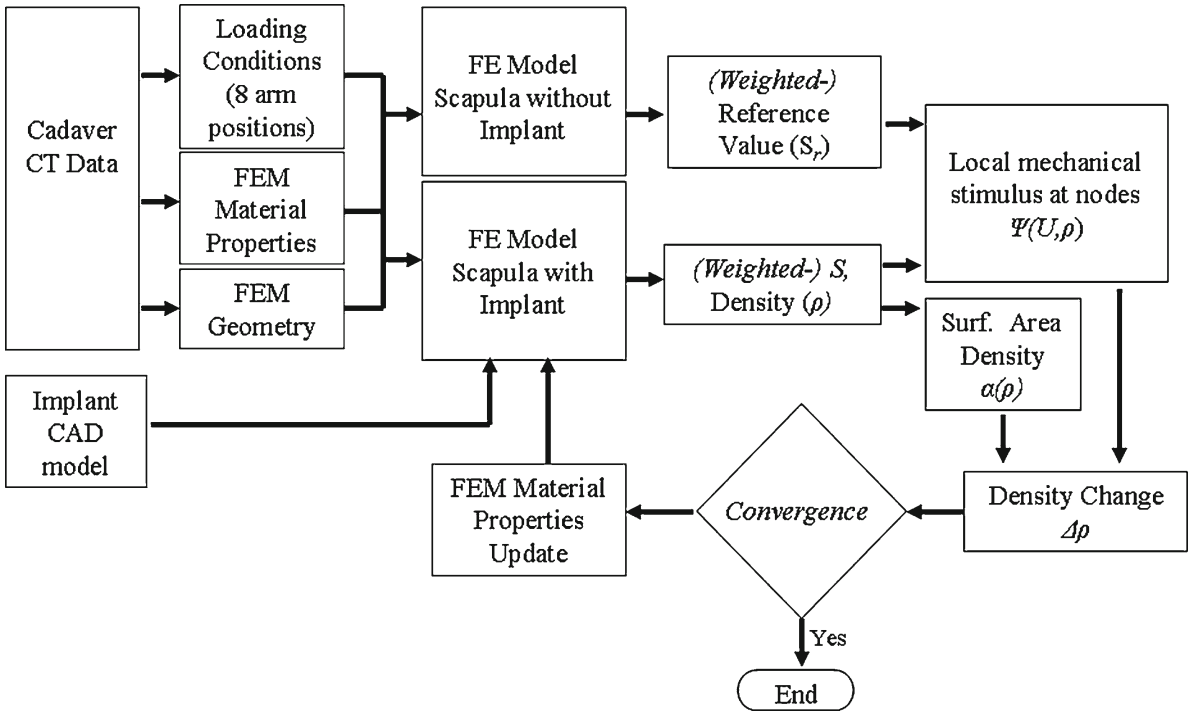

remodelling algorithm is terminated when the absolute density change did not change more than $0.005 \mathrm{~g} / \mathrm{cm}^{3}$ per element per increment $(\tau \Delta t)$. This convergence criterion $(C)$ can be written as

$C=\frac{\left(\sum_{i=1}^{n}\left\|\Delta \rho_{i}\right\|\right)}{n \cdot(\tau \Delta t)}$

and therefore, $C$ is independent of the chosen increment size (i.e. time step) and its dimensions are density units per units of the 'simulation time scale'. The results of the two considered cases, bonded and loose interface, are given in terms of the average density in the glenoid vault, histograms of the element densities and the largest maximum and minimum principal strains during the bone adaptation. In case of a loose interface, bone-implant interface micromotions are also assessed at the first and last iteration of the bone remodelling simulation. The applied bone remodelling scheme is shown in Fig. 2.

\section{Results}

Two FE models of two extreme scenarios after a TSA were created and solved. In the first one, an ideal case in which bone ingrowth occurred in the complete bone-implant interface shortly after surgery is simulated (i.e. model with bonded interface). In a second model, the worst possible scenario is considered, when the bone-implant interface is completely loose due to absence of bone ingrowth (i.e. model with loose interface). In both cases, the bone adaptation process reached the desired convergence criterion after $\sim 50$ iterations.

Bone adaptation after TSA is presented in terms of the density per element during the bone remodelling. Density changes mostly happened during the first 10 iterations; however, the adaptation of the bone continued at a slower rate through the complete simulation. Cross-sections of the glenoid through the adaptation process for a fully bonded interface are presented in Fig. 3 and for a loose interface in Fig. 4. Additionally, change in the frequency of the element density values, i.e. density histograms, for both models and through the complete simulation are presented in Fig. 5.

\subsection{General changes after TSA}

Independently of the interface condition, the implantation of the cementless metal-backed glenoid component produced a significant change in the distribution of the glenoid density. Extensive bone resorption in the cancellous bone at the posterior and superior portions of the interface was evident after the first ten iterations. Bone apposition was mainly seen in the posterior cortical wall, the superior and inferior cortical rim, and around the proximal section of the screw in the glenoid neck. These trends continued throughout the complete simulated time, increasing the load transfer trough the rigid central screw and a large section of the cortical wall, shielding the cancellous bone at the bone-implant interface. Figure 6 shows the relative change of bone density with respect to the original condition before implantation.

\subsection{Particular changes depending on the interface condition}

In addition to the mentioned changes, bone apposition in cancellous bone next to the inferior-anterior region of the interface occurred when the interface was considered bonded, see Fig. 6. Bone apposition in case of a loose interface was more limited, despite some increment in bone density in the superior region of the interface. 
Fig. 3 Snapshots of the elements' bone density changes during the bone adaptation process when the bone-implant interface is considered fully bonded. No post-processing filtering was used to smooth the outcome of the models

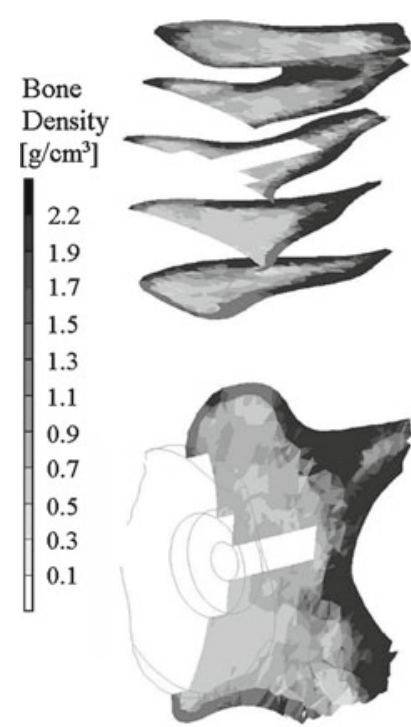

Time Inc.: 0

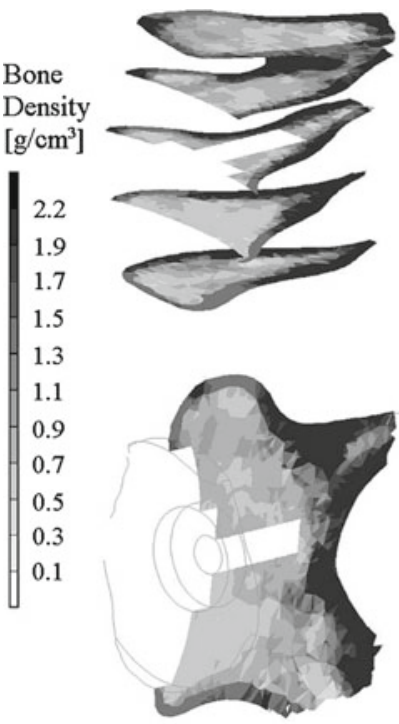

Time Inc.: 0
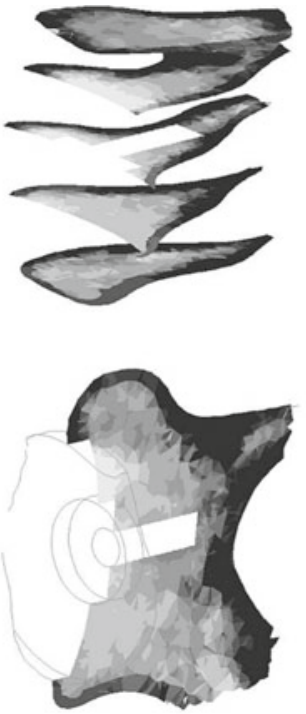

Time Inc.: 10
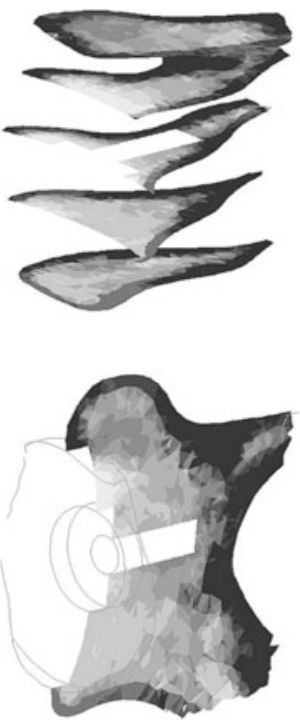

Time Inc.: 10

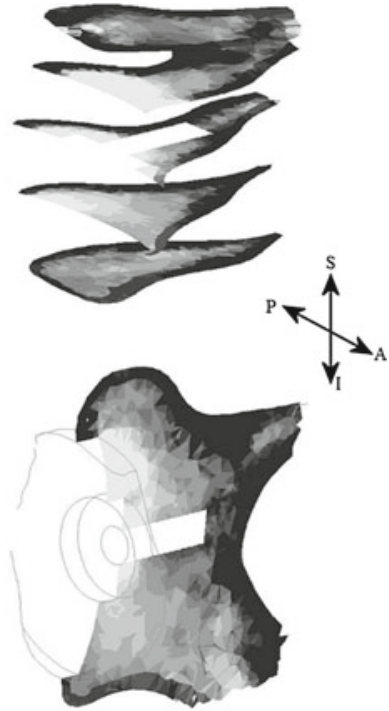

Time Inc.: 50

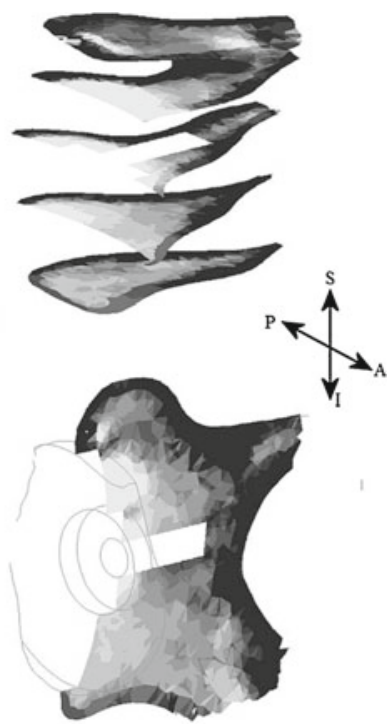

Time Inc.: 50

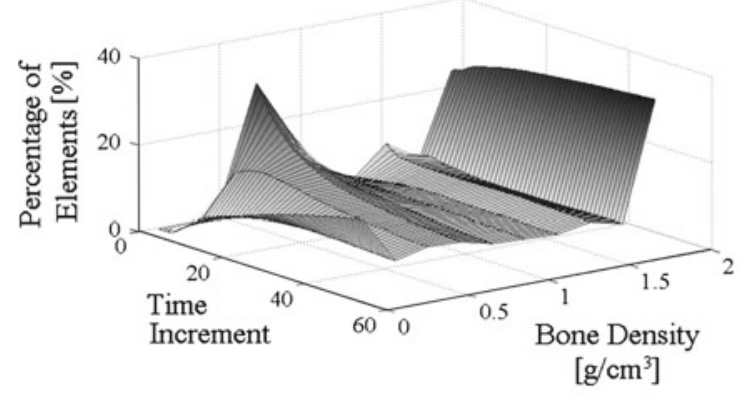

Loose Interface

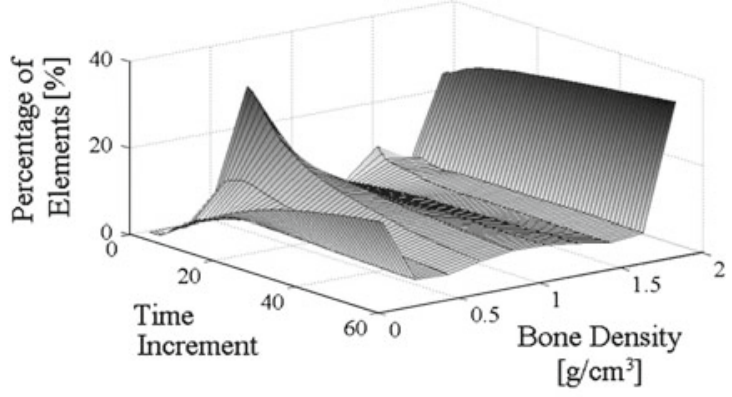

Bonded Interface

Fig. 5 Percentage of elements in the glenoid according to their (bone) density values and its change during the complete bone remodelling simulation. Surfaces computed from the density histograms in each iteration 

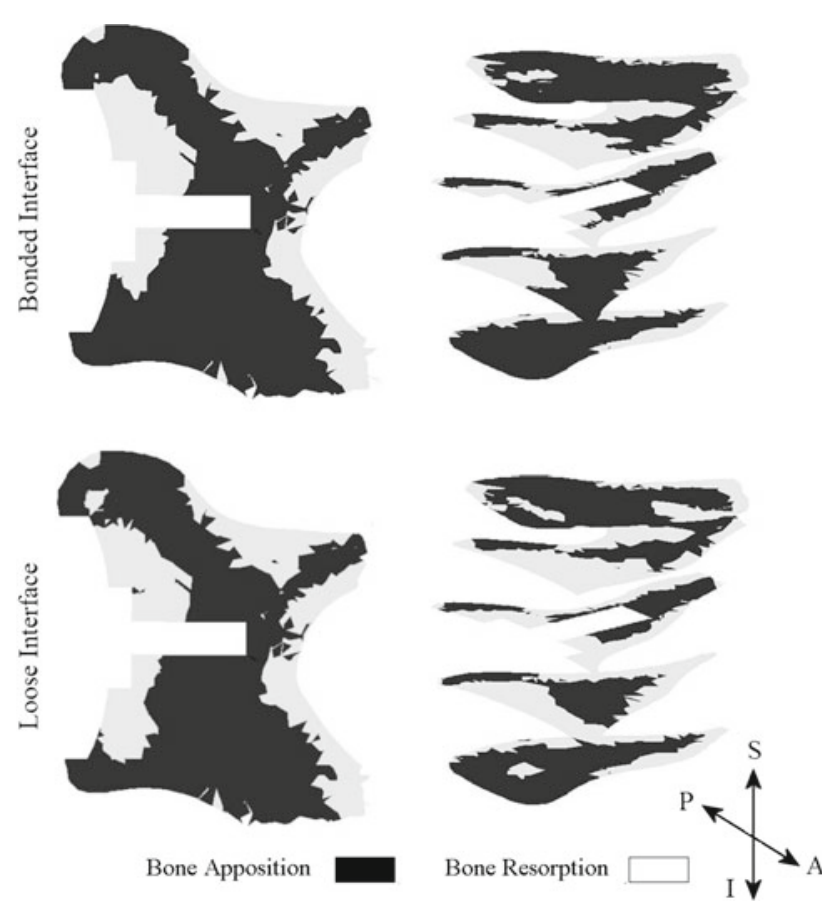

Fig. 6 Relative change of the bone density with respect to the values before surgery (TSA)
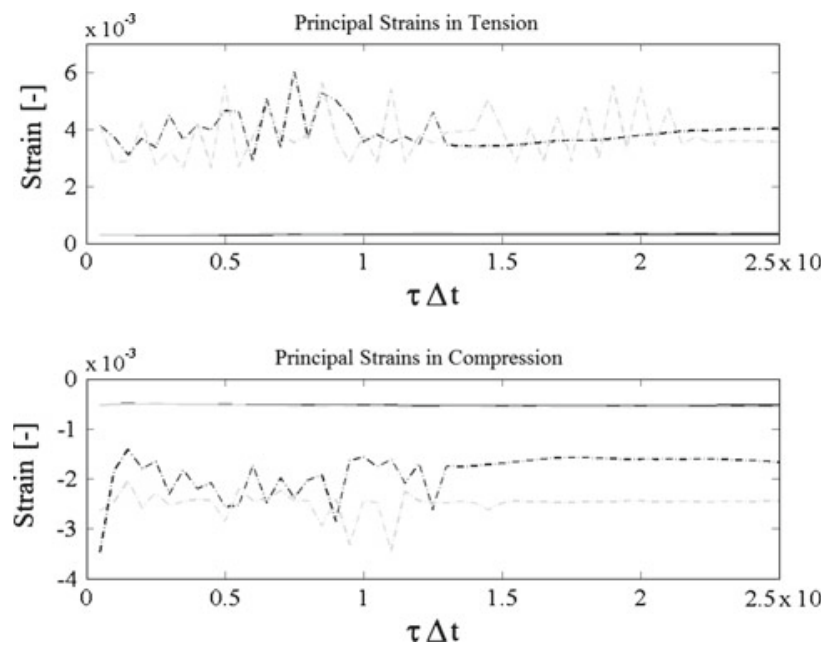

Fig. 7 Principal strains in the glenoid when the bone-implant interface is considered bonded (black) an loose (grey), largest absolute value (dot line) and 90th percentile (continuous line), during the bone adaptation process in the glenoid

\subsection{Strains during the remodelling process}

Maximum and minimum principal strains were calculated during the bone adaptation. Figure 7 shows the largest principal strains during the bone remodelling process. Strain peaks reaching $0.6 \%$ in tension and $0.3 \%$ in compression occurred at interface elements, but during a large part of the simulation, principal strains in most of the glenoid never exceeded $0.1 \%$ in either tension or compression.

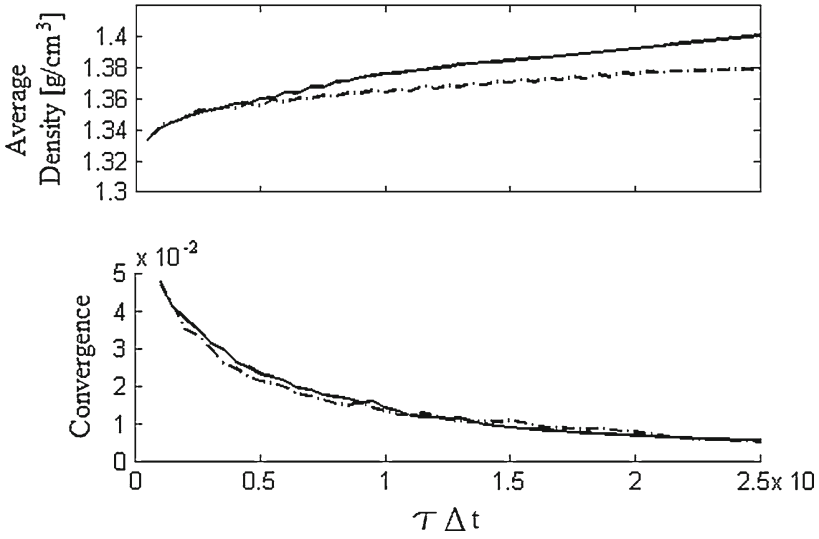

Fig. 8 Above average glenoid density and convergence of the FE models when bone-implant interface is considered fully bonded (continuous line) or completely loose (dotted line). Below convergence of the model as absolute density change per element per time increment $(\tau \Delta t)$

\subsection{The average glenoid density and the convergence} of the model

Regardless of an important bone resorption next to the boneimplant interface, the average glenoid density increased after TSA, see Fig. 8. The average density in the glenoid was always larger for the model with a fully bonded interface, and the difference increased with time. Independent of the interface conditions, the bone adaptation was still ongoing, but at a much lower pace when the convergence $(C)$ criterion was reached.

\subsection{Mechanical long-term stability of the implant}

Bone-implant interface micromotions, as a measure of the implant's mechanical stability in case of a loose interface, did not change during the bone adaptation process. The maximum bone-implant interface micromotions were always below $10 \mu \mathrm{m}$, and their median value did not change significantly $(\mathrm{p}>0.1)$ after bone adaptation.

\section{Discussion}

A number of studies have already pointed towards a stress shielding phenomenon and possible bone adaptation around glenoid components. They predict strengthening of parts of the cortical bone and weakening of the cancellous bone based on the mechanical loading conditions of bone right after TSA (Pelletier et al. 2008; Maurel et al. 2002; Gupta et al. 2004a; Orr et al. 1988; Stone et al. 1999).

The presented 3D FE models of a scapula with a cementless glenoid component, with material properties, geometry and physiological loading conditions based on a single cadaver (Suárez et al. 2009a,b), were used to predict the bone adaptation in the glenoid bone after a TSA. Two extreme 
conditions were considered: when the bone-implant interface is entirely bonded, due to an idealistic $100 \%$ bone ingrowth, and when the same interface is completely loose, due to total absence of bone ingrowth. Beside the FE models, the simulation included a quantitative formulation of the time-dependent bone adaptation process previously described by Huiskes et al. (1987) and Weinans et al. (1993). A nodal-based scheme was used to implement the bone adaptation process and avoid numerical instabilities that are inherent in element base schemes (Jacobs et al. 1995). The changes in the glenoid bone after TSA were assessed in terms of bone density, locally and throughout the entire glenoid vault.

The bone adaptation process depends, of course, on the loading conditions of the scapula and implant. Hence, an accurate representation of the loading history is very important to achieve realistic results (Carter et al. 1987). In the presented simulation, the contribution to the remodelling signal $(S)$ of muscles and joint reaction forces at each one of eight arm positions was weighted according to how often the arm is in that position during activities of daily life.

\subsection{General results}

Although bone ingrowth into the porous coating of the component's metal back was not actually included, the two bone-implant interface conditions considered in this study (completely bonded or loose interface) should give a range of the possible bone remodelling in the glenoid after TSA, regardless of the actual state of the interface. However, the resulting density distribution and average density were not extremely different when the interface condition changed. A small difference on bone remodelling using either constant or variable interface conditions, but around humeral components, was also found by other authors (Fernandes et al. 2002). Nevertheless, a variable interface condition due to bone ingrowth may have generally a considerable influence on the bone remodelling around an implant, as shown by Folgado et al. (2009).

In the presented simulations, using either bonded or loose interface, an extended cancellous bone resorption occurred next to the bone-implant interface, while there was bone apposition in the proximal section of the screw at the glenoid neck and some sections of the cortical and adjacent bone. In particular, a bonded interface allowed loading of the inferior and anterior regions of the interface, and subsequently, the local bone density at these regions increased. On the other hand, when a loose interface was simulated, bone resorption was predominant in the same anterior and inferior portions of the glenoid.

The increment of the average bone density in the glenoid is an unexpected result of this study, especially considering that bone remodelling normally leads to an extensive bone loss, as it is the case when a femoral component with rigid stem is inserted during a hip arthroplasty (HA). The present model predicts also a considerable bone loss around the glenoid; however, a gain in density in the mentioned sections of the glenoid pushed the average density up. The gain in those parts of the scapula was only limited by the maximal bone density allowed. Regardless of the unexpected increment in the average density, the result is plausible: differently to the case in HA, where the stem shields both cortical and cancellous bone to the loads, the metal-backed glenoid component finds is best support in the cortical ring around the glenoid, and therefore, that cortical bone gains density while the cancellous bone near the interface and in the middle of the glenoid is shielded and lost density, as it is predicted by the present model.

Recent follow-up studies with metal-backed glenoid components have indicated a high prevalence of radiolucent lines around the glenoid implants: $83 \%$ after 2 years in cemented components (Tammachote et al. 2007) and 45\% for cementless components (Martin et al. 2005). Stress shielding might be a reason, or at least a contributing factor, for the progressive radiolucency that is observed at the boneimplant interface. Stress shielding will concert together with osteolysis associated with the presence of PE or PMMA particles. The predicted progressive bone resorption at the superior region of the interface in this study agrees to what has been found in clinics in the mid and long term for similar components, see for instance the radiographs in Martin et al. (2005) and Clement et al. (2010).

Despite the extensive change in the bone density distribution in the glenoid, the fixation screw was able to keep the implant in position with minimum interface micromotions. However, we must highlight that the screw model did not include fatigue of its body and weakening of its fixation in time; both factors are certainly present in the actual prosthetic joint. The result is comparable with some clinical cases, in which large radiolucent lines are presented around the component, but it is considered stable at the moment of the follow-up (Martin et al. 2005).

Minimal interface gap and micromotions are considered to be requirements for bone ingrowth around an implant (Harris et al. 1983; Kienapfel et al. 1999). However, these are not exclusive conditions. It is not likely that bone ingrowth succeeds in places where bone resorption is present, even when the gap and micromotions are negligible, i.e. in a bonded interface. This might be a reason why, even in retrievals of successful glenoid components, bone ingrowth never covers the totality of the porous coating of this type of implant.

Despite an accurate prediction of the strain in trabeculae not being possible due to the homogenization of the cancellous bone, it was calculated to study how the overall mechanical loading of bone changes during the bone adaptation process. Initially, regions with the largest principal strains, either in compression or tension, were limited to places with stress concentrators at the interface (e.g. sharp 
edges of the metal back). Later, elements at the interface and with the minimum density value reached the same strain level. Regardless of strain peaks during the bone adaptation process, principal strains in the complete glenoid were smaller than yield values reported for bone tissue, $0.62 \%$ in tension and $-1.04 \%$ in compression (Bayraktar et al. 2004).

\subsection{Bone adaptation time scale and limitations}

The results are presented using the simulation time scale ( $\tau \Delta t)$. The constant $\tau$ can be obtained through direct comparison between the simulation outcome and clinical images that are acquired during a follow-up of a patient or animal with the same prosthesis. Evaluation of $\tau$ and comparison between models and in vivo experiments have been done for some femoral components used in hip arthroplasty $\left(\tau=129.6 \mathrm{~g} / \mathrm{mm}^{2}(\mathrm{~J} / \mathrm{g})\right.$ months, Weinans et al. 1993). Nevertheless, the bone adaptation rate seems to be site-dependant. If this value is used in this simulation, the actual time for the bone remodelling here presented would be around one week, which is impossible. Therefore, new experiments or a clinical follow-up of a patient using the same prosthesis would be necessary to evaluate $\tau$ correctly.

Next to $\tau, z$ is another arbitrary constant in the applied bone remodelling algorithm. Its small value in comparison with other studies (van Rietbergen et al. 1993; Weinans et al. 1993) means a negligible 'dead' zone. A small $z$ could slow down the convergence rate and modify the evaluation of $\tau$, but the final outcome of the remodelling algorithm is not expected to change.

Bone ingrowth was not actually included in the model and a variable interface condition may affect the bone adaptation process in some cases (e.g. other implant design or loading conditions). Therefore, future simulations of a TSA including bone ingrowth and which are able to change the interface conditions will be useful to generalize results and conclusions.

Adaption of the scapula geometry to the loading conditions was not included in this study, limiting the bone adaption exclusively to the change in local density. The scapula, from which geometry, bone material properties and loading conditions were obtained, was considered healthy (Veeger et al. 1991), and this may have implications for the presented results. Bone adaptation is known to be dependent on the initial conditions and loading conditions, thus the outcome of the simulation is limited to the analysed case. However, qualitative features of the outcome, as the bone apposition around the metal screw and the bone loss next to the bone-implant interface, are likely to happen also in a low-quality bone.

\section{Conclusions}

Evaluation of the mechanically induced bone adaptation after TSA was achieved using a (cadaver-specific) 3D FE model of a scapula with a cementless metal-backed glenoid component in combination with a site-specific and node-based bone remodelling algorithm.

Although the simulation results are not presented in an actual time scale (e.g., months, years), this work confirmed a general bone adaptation to the new loading conditions, when a cementless metal-backed glenoid component is implanted in the scapula. These new conditions produced a gain in the average glenoid bone density, but an important cancellous bone loss at the bone-implant interface. While this adaptation did not affect the long-term mechanical stability of the glenoid component, in terms of the bone-implant interface micromotions, it is important to highlight that other factors such as fatigue of the screw(s), bone resorption due to strange-body reactions, etc., can also undermine the longterm stability of the implant.

The success of bone ingrowth in the metal back of the glenoid component had a limited influence on the general adaptation of the glenoid after implantation. An idealistic bone ingrowth in the complete bone-implant interface, simulated as a fully bonded interface, did not avoid an extended bone resorption at the glenoid due to the mechanically induced adaptation.

Despite the fact that the presented simulation has successfully included part of the complexity of the bone adaptation around a cementless glenoid component, generalization of the presented results is still necessary. Further steps include evaluation of different implant designs and a comparison to clinical follow-up studies, or in vivo experiments, that evaluate the time frame in which the bone adaptation in the glenoid occurs. These further steps will allow predicting the long-term mechanical stability of new designs before clinical trails and the possible bone adaptation around them.

Acknowledgments The authors thank to Prof. Piet M. Rozing and Dr. Edward R. Valstar for their valuable feedback and discussions during the elaboration of this manuscript. This research was supported by a long-term grant of the Dutch Arthritis Association.

Open Access This article is distributed under the terms of the Creative Commons Attribution Noncommercial License which permits any noncommercial use, distribution, and reproduction in any medium, provided the original author(s) and source are credited.

\section{References}

Bayraktar HH, Morgan EF, Niebur GL, Morris GE, Wong EK, Keaveny TM (2004) Comparison of the elastic and yield properties of human femoral trabecular and cortical bone tissue. J Biomech 37(1):27-35

Bohsali KI, Wirth MA, Rockwood JCA (2006) Complications of total shoulder arthroplasty. J Bone Joint Surg Am 88(10):2279-2292

Boissonnat JD (1988) Shape reconstruction from planar cross sections. Comput Vis Graph Image Process 44(1):1-29

Botha CP, Post FH (2008) Hybrid scheduling in the deVIDE dataflow visualisation environment. In: Hauser $\mathrm{H}$, Strassburger $\mathrm{S}$, Theisel 
H (eds) Simulation and visualization 2008 (SimVis 2008). SCS Publishing House e.V., Magdeburg pp 309-322

Carter DR, Fyhrie DP, Whalen RT (1987) Trabecular bone density and loading history: regulation of connective tissue biology by mechanical energy. J Biomech 20(8):785-794

Clement ND, Mathur K, Colling R, Stirrat AN (2010) The metal-backed glenoid component in rheumatoid disease: eight- to fourteen-year follow-up. J Shoulder Elbow Surg 19(5):749-756

Coley B, Jolles BM, Farron A, Aminian K (2008) Arm position during daily activity. Gait Posture 28(4):581-587

Couteau B, Mansat P, Estivalezes E, Darmana R, Mansat M, Egan J (2001) Finite element analysis of the mechanical behavior of a scapula implanted with a glenoid prosthesis. Clin Biomech (Bristol, Avon) 16(7):566-575

Fernandes P, Folgado J, Jacobs C, Pellegrini V (2002) A contact model with ingrowth control for bone remodelling around cementless stems. J Biomech 35(2):167-176

Folgado J, Fernandes P, Jacobs C, Pellegrini V (2009) Influence of femoral stem geometry, material and extent of porous coating on bone ingrowth and atrophy in cementless total hip arthroplasty: an iterative finite element model. Comput Methods Biomech Biomed Eng 12(2):135-145

Frost H (1964) The laws of bone structure. C.C. Thomas, Springfield

Frost HM (1988) Vital biomechanics: proposed general concepts for skeletal adaptations to mechanical usage. Calcif Tissue Int 42(3): $145-156$

Gupta S, van der Helm FC, van Keulen F (2004a) The possibilities of uncemented glenoid component - a finite element study. Clin Biomech (Bristol, Avon) 19(3):292-302

Gupta S, van der Helm FC, van Keulen F (2004b) Stress analysis of cemented glenoid prostheses in total shoulder arthroplasty. J Biomech 37(11):1777-1786

Harris WH, White JRE, McCarthy JC, Walker PS, Weinberg EH (1983) Bony ingrowth fixation of the acetabular component in canine hip joint arthroplasty. Clin Orthop Relat Res 176:7-11

Hart R (2001) Bone modelling and remodelling: theories and computation. In: Cowin SC (ed) Bone mechanics handbook, vol 1. 2nd edn, CRC Press, Boca Raton

Hollister SJ, Brennan JM, Kikuchi N (1994) A homogenization sampling procedure for calculating trabecular bone effective stiffness and tissue level stress. J Biomech 27(4):433-444

Huiskes R, Weinans H, Grootenboer HJ, Dalstra M, Fudala B, Slooff TJ (1987) Adaptive bone-remodelling theory applied to prosthetic-design analysis. J Biomech 20(11-12):1135-1150

Jacobs CR, Levenston ME, Beaupre GS, Simo JC, Carter DR (1995) Numerical instabilities in bone remodeling simulations: the advantages of a node-based finite element approach. J Biomech 28(4): 449-459

Kalouche I, Crépin J, Abdelmoumen S, Mitton D, Guillot G, Gagey O (2010) Mechanical properties of glenoid cancellous bone. Clin Biomech (Bristol, Avon) 25(4):292-298

Kienapfel H, Sprey C, Wilke A, Griss P (1999) Implant fixation by bone ingrowth. J Arthroplasty 14(3):355-368

Lacroix D, Murphy LA, Prendergast PJ (2000) Three-dimensional finite element analysis of glenoid replacement prostheses: a comparison of keeled and pegged anchorage systems. J Biomech Eng 122(4):430-436

Lehtinen J, Tingart M, Apreleva M, Warner J (2004) Total, trabecular, and cortical bone mineral density in different regions of the glenoid. J Shoulder Elbow Surg 13(3):344-348

Martin RB (1972) Effects of geometric feedback in development of osteoporosis. J Biomech 5(5):447-455

Martin RB (1984) Porosity and specific surface of bone. Crit Rev Biomed Eng 10(3):179-222
Martin SD, Zurakowski D, Thornhill TS (2005) Uncemented glenoid component in total shoulder arthroplasty. Survivorship and outcomes. J Bone Joint Surg Am 87(6):1284-1292

Maurel N, Diop A, Grimberg J, Elise S (2002) In vitro biomechanical analysis of glenoids before and after implantation of prosthetic components. J Biomech 35(8):1071-1080

McKellop H, Clarke I, Markolf K, Amstutz H (1981) Friction and wear properties of polymer, metal, and ceramic prosthetic joint materials evaluated on a multichannel screening device. J Biomed Mater Res 15(5):619-653

Orr TE, Carter DR, Schurman DJ (1988) Stress analyses of glenoid component designs. Clin Orthop Relat Res 232:217-224

Pelletier MH, Langdown A, Gillies RM, Sonnabend DH, Walsh WR (2008) Photoelastic comparison of strains in the underlying glenoid with metal-backed and all-polyethylene implants. J Shoulder Elbow Surg 17(5):779-783

Popinet S, Jones T (2006) GTS, GNU Triangulated Surface Library. http://gts.sourceforge.net. Accessed 10 June 2006

Rice JC, Cowin SC, Bowman JA (1988) On the dependence of the elasticity and strength of cancellous bone on apparent density. J Biomech 21(2): 155-168

Schaffler MB, Burr DB (1988) Stiffness of compact bone: effects of porosity and density. J Biomech 21(1):13-16

Stone KD, Grabowski JJ, Cofield RH, Morrey BF, An KN (1999) Stress analyses of glenoid components in total shoulder arthroplasty. J Shoulder Elbow Surg 8(2):151-158

Suárez DR, van der Linden JC, Valstar ER, Broomans P, Poort G, Rozing PM, van Keulen F (2009) Influence of the positioning of a cementless glenoid prosthesis on its interface micromotions. Proc Inst Mech Eng H 223:795-804

Suárez DR, Valstar ER, van der Linden JC, van Keulen F, Rozing PM (2009) Effect of rotator cuff dysfunction on the initial mechanical stability of cementless glenoid components. Med Biol Eng Comput 47(5):507-514

Tammachote N, Sperling JW, Berglund LJ, Steinmann SP, Cofield RH, An KN (2007) The effect of glenoid component size on the stability of total shoulder arthroplasty. J Shoulder Elbow Surg 16(3 Suppl):S102-S106

Torchia ME, Cofield RH, Settergren CR (1997) Total shoulder arthroplasty with the Neer prosthesis: long-term results. J Shoulder Elbow Surg 6(6):495-505

van der Helm FC (1994) A finite element musculoskeletal model of the shoulder mechanism. J Biomech 27(5):551-569

van der Helm FC, Veeger HE, Pronk GM, Vander Woude LH, Rozendal RH (1992) Geometry parameters for musculoskeletal modelling of the shoulder system. J Biomech 25(2):129-144

van Rietbergen B, Huiskes R, Weinans H, Sumner DR, Turner TM, Galante JO (1993) ESB research award 1992. The mechanism of bone remodeling and resorption around press-fitted THA stems. J Biomech 26(4-5):369-382

van Rietbergen B, Huiskes R (2001) Elastic constants of cancellous bone. In: Cowin $\mathrm{S}$ (ed) Bone mechanics handbook. 2nd edn, CRC Press, Boca Raton

Veeger HE, van der Helm FC, Vander Woude LH, Pronk GM, Rozendal RH (1991) Inertia and muscle contraction parameters for musculoskeletal modelling of the shoulder mechanism. J Biomech 24(7):615-629

Weinans H, Huiskes R, van Rietbergen B, Sumner DR, Turner TM, Galante JO (1993) Adaptive bone remodeling around bonded noncemented total hip arthroplasty: a comparison between animal experiments and computer simulation. J Orthop Res 11(4):500 513

Wirth MA, Rockwood CA (1996) Complications of total shoulderreplacement arthroplasty. J Bone Joint Surg Am 78(4):603-616 
Yeh LR, Kwak S, Kim YS, Chou DS, Muhle C, Skaf A, Trudell D, Resnick D (1998) Evaluation of articular cartilage thickness of the humeral head and the glenoid fossa by $\mathrm{mr}$ arthrography: anatomic correlation in cadavers. Skeletal Radiol 27(9):500-504
Zhang Y, Ahn P, Fitzpatrick D, Heiner A, Poggie R, Brown $\mathrm{T}$ (1999) Interfacial frictional behavior: cancellous bone, cortical bone, and a novel porous tantalum biomaterial. J Musculoskelet Res 3(4):245-251 